\title{
Gene Repression Paradigms in Animal Cells
}

\author{
L. Lande-Diner, J. Zhang, T. Hashimshony, A. Goren, \\ I. KeShet, AND H. CEDAR \\ Department of Cellular Biochemistry, Hebrew University, Jerusalem, 91120 Israel
}

In simple organisms with relatively small genomes almost all of the genes are expressed in every cell. Silenced genes are usually controlled by specific repressor proteins that recognize and bind cis-acting elements in the vicinity of the gene, thereby preventing transcription. This organization contrasts dramatically with the program of repression in higher multicellular organisms. In these cells, one observes a bimodal pattern of expression. Housekeeping genes are transcribed constitutively while other genes are tissue specific and thus turned off in most cell types. These repressed genes probably make up $>60 \%$ of the genome. Although repression mechanisms for individual genes have been studied in animal cells, little is known about the overall organization and programming of gene silencing.

One of the key mechanisms involved in gene repression in animal cells is DNA methylation. The genome itself appears to be modified in a bimodal pattern with most of the DNA being methylated, while CpG islands at the 5' end of housekeeping genes are constitutively unmethylated. Genes that do not contain $\mathrm{CpG}$ islands are generally repressed by the presence of DNA methylation in the vicinity of their promoter sequences. It should be noted that, unlike the genetic information contained in the DNA sequence, the methylation profile is not inherited from the germ line (Kafri et al. 1992).

Almost all DNA methylation coming from the germ cells is erased from the genome in the morula, and the bimodal pattern is then reestablished anew in each individual at the time of implantation (Kafri et al. 1992). At this stage the embryo expresses powerful de novo methylases capable of modifying the entire genome (Okano et al. 1999). In parallel, cis-acting sequences within $\mathrm{CpG}$ islands are recognized by specific trans-acting factors that serve to protect these regions from modification (Brandeis et al. 1994; Macleod et al. 1994). Once established, this bimodal pattern is preserved intact following each round of cell division through the action of a maintenance methylase that copies the $\mathrm{CpG}$ methylation profile present on the native DNA strand once the replication fork has passed (Cedar 1988). It should be noted that this repression mechanism operates without the need to recognize or identify specific gene sequences and, in this sense, is truly global. In addition, this represents a genuine epigenetic regulatory mechanism because it is preprogrammed to be preserved as part of the genome sequence itself.

\section{METHYLATION MODELS HISTONE MODIFICATION}

In order to better understand how methylation contributes to gene repression, we designed an experiment in which the methylation state of a transgene could be controlled in vivo, and then used these animals to examine the effect of DNA methylation on chromatin structure (Fig. 1a). The test gene (Hashimshony et al. 2003) was composed of a normal human $\beta$-globin sequence driven by its own natural minimal promoter placed near a 120 base pairs (bp) segment (island element [IE]) of the mouse Aprt $\mathrm{CpG}$ island that has been shown to protect against de novo methylation at the time of implantation (Siegfried et al. 1999). This IE is actually part of a cassette flanked by two LoxP sites. When this mouse is crossed with mice that express Cre from early stages of embryogenesis (Lallemand et al. 1998), the presence of this trans-acting factor causes the IE to be deleted prior to implantation, and as a result the nearby $\beta$-globin promoter region undergoes de novo methylation, which is then maintained in all cells of the offspring.

Alternatively, when the adult mouse is mated with animals that express an interferon inducible Cre gene (Mx) (Kuhn et al. 1995), the IE element remains intact at the time of implantation, thus protecting against de novo methylation in the promoter region. When adult mice are then treated with the inducer, newly synthesized Cre protein brings about deletion of the IE, while still leaving the promoter region in its unmethylated state. Using these crosses, it is thus possible to generate identical transgenic mice that carry either a methylated or nonmethylated promoter. Previous studies (Siegfried et al. 1999) have demonstrated that while the level of transcription is low in both animals, the presence of DNA methylation at the promoter brings about a 50-100-fold inhibition of $\beta$ globin basal expression in many different cell types.

We next asked whether DNA methylation influences chromatin structure at the level of local histone modification. To this end, we isolated lymphoid cells from the spleen and prepared mononucleosomes that were then subjected to chromatin immunoprecipitation (ChIP) analysis using antibodies to acetylated histones (Fig. 1b). The bound and input DNA were then used to assay nucleosomes in the $\beta$-globin promoter region by means of semiquantitative polymerase chain reaction (PCR). As a con- 
a.
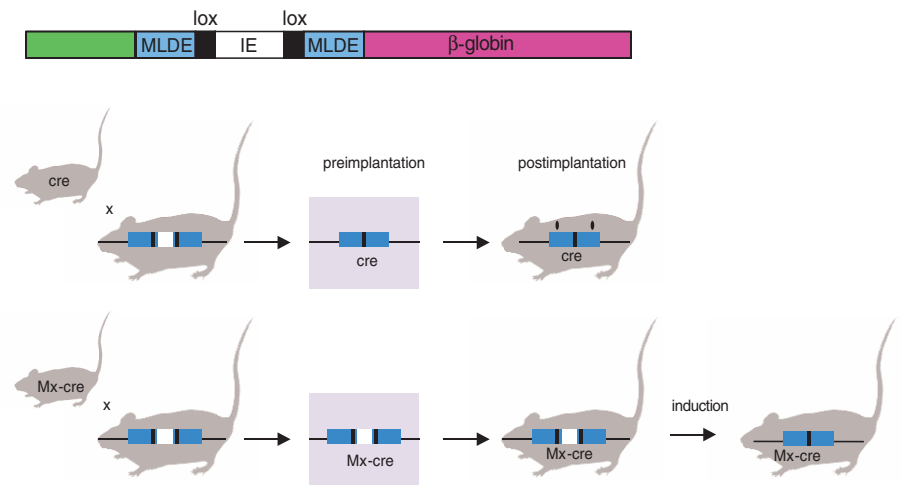

b.

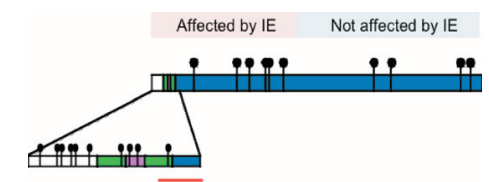

C.
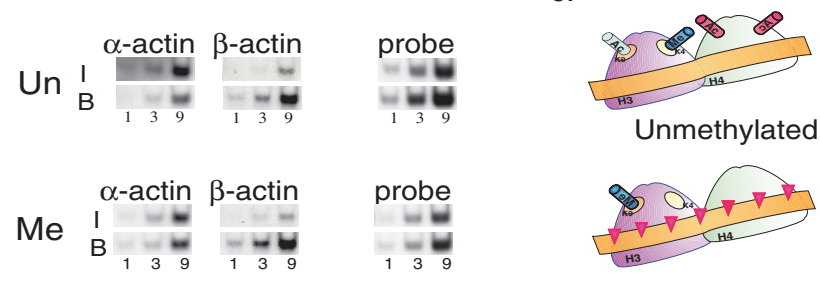

Methylated

Figure 1. (a) Programmed transgene methylation. The transgene construct (not shown to scale) used in this study is made up of human $\beta$-globin $(H B B)$ sequences (pink), including a 48-bp minimal promoter, two 30-bp MLDE sequences (blue), two 34-bp loxP elements (black), the 120-bp island element (IE; white), and 100 bp of flanking buffer DNA sequences (green). Mouse bML120 $\beta$ contains a single copy of this transgene. To generate mice carrying transgenes identical in sequence but either methylated or unmethylated, the loxP-flanked island element was deleted by mating carriers with two different Cre-expressing lines. In the first line, Cre is expressed before implantation. In mice carrying this construct (Cre mice), the island element is deleted before the wave of de novo methylation, and surrounding $\mathrm{CpG}$ sites thus become methylated (black circles). The second Cre-expressing line carries interferon-inducible cre. In these mice (Mx-Cre), the island element remains present during implantation and protects adjacent regions from methylation. By treating adult mice with polyI-polyC, Cre activity is induced and the island element is removed, generating an unmethylated version of the transgene flanked by loxP sites (Hashimshony et al. 2003). (b) Mononucleosomes were prepared from spleens taken from mice with either the methylated (Me) or the unmethylated (Un) ML ${ }^{\beta}$ transgene (Hashimshony et al. 2003) and subjected to ChIP analysis using antibody to acetylated H4. The map shows the locations of CpG residues (black circles) in the construct. All of the $\mathrm{CpG}$ sites were constitutively modified in mice carrying the methylated transgene. In mice with the unmethylated transgene, the region whose methylation is affected by the IE is $>90 \%$ unmodified. Input (I) and bound (B) DNA fractions were used for quantitative PCR on samples of 1,3 , or $9 \mu \mathrm{l}$ using primer sets specific for the region shown on the map (red line). The input DNA was diluted at least 1:100 to allow comparison to bound DNA. For each ChIP preparation, $\alpha$-actin was assayed as a negative control and $\beta$ actin as a positive control (Hashimshony et al. 2003). (c) The histone modification pattern and DNA structure (ribbon) of the unmethylated and methylated (red triangles) transgene. Unmethylated DNA is packaged in an open configuration, where histone $\mathrm{H} 3$ is acetylated in $\mathrm{K} 9$ and methylated in $\mathrm{K} 4$, and histone $\mathrm{H} 4$ is acetylated. Methylated DNA is packaged in a closed chromatin structure, where histone $\mathrm{H} 3$ is methylated in the K9 residue and demethylated in lysine 4, and histone H4 is deacetylated.

trol, we first showed that an active gene like $\beta$-actin is indeed enriched for Ac-H3, while an inactive gene such as $\alpha$ actin is depleted for this modification. Interestingly, the unmethylated $\beta$-globin promoter on the transgene was also found to be in an active acetylated conformation. In contrast, when methylated, this same region is depleted for histone acetylation. By analyzing additional transgene founders, we found that this effect is highly reproducible and clearly differentiates between methylated and nonmethylated DNA over a defined region surrounding the IE, but not in areas unaffected by this element. Additional experiments employing antibodies to other histone modifications showed that the presence of DNA methylation has a big influence on histone structure, causing chromatin to be packaged with nucleosomes that have deacetylated histones $\mathrm{H} 3$ and H4, which are also unmethylated at histone H3(Lys4). It thus appears that DNA methylation represents an epigenetic mark that causes DNA to be packaged in "closed" nucleosomes after every round of replication.

\section{GENE REPRESSION PATTERNS}

While these studies clearly demonstrate that DNA methylation serves as a global repressor of gene structure and activity, it must be remembered that the genome uti- 
lizes additional gene repression mechanisms, as well. In order to evaluate the relative contribution of DNA modification to the overall process of gene silencing, it is thus necessary to remove methyl moieties from the DNA, and then determine how this effects gene expression. To this end, we prepared primary mouse embryo fibroblasts that are Dnmt1 ${ }^{-}$(Li et al. 1992) on a p53- background. Since the amount of Dnmt1 in these cells is limiting, DNA methylation is diluted out, and after about ten generations the cells have lost $95 \%$ of their methyl groups as shown by nearest neighbor analysis (Gruenbaum et al. 1981).

In order to evaluate the effect of this undermethylation on gene expression, we prepared RNA from $\mathrm{p} 53^{-/-}$control cultures and from $\mathrm{p} 53^{-/}$Dnmt1 ${ }^{-/}$cells and hybridized to microarray chips (Affymetrix) containing 22,000 mouse gene cDNA segments. Since DNA methylation is thought to work through its effect on histone acetylation (Razin 1998), we also examined the expression pattern induced by treatment of these cells with Trichostatin A (TSA), a potent inhibitor of histone deacetylation. This analysis revealed the presence of four distinct gene repression paradigms: genes that are unaffected by TSA and Dnmt1 $1^{-/}$, genes induced by TSA treatment alone, genes induced by demethylation alone, and genes that could be expressed only following a combination of demethylation and TSA treatment.

\section{GENE STRUCTURE PATTERNS}

We next addressed ourselves to understanding the relationship between these expression paradigms and gene structure. To this end, we chose about 40 tissue-specific genes representative of the four categories and analyzed the methylation state and histone modification pattern in their promoter regions. The DNA methylation status of each gene was determined by methylated DNA immunoprecipitation (mDIP), a new technique that utilizes an antibody specific to $5 \mathrm{mC}$ (Reynaud et al. 1992) to identify methylated sequences by PCR analysis. Histone modifications, including Ac-H4, Ac-H3, 2me-H3(K9), and me$\mathrm{H} 3(\mathrm{~K} 4)$, were assessed by ChIP. In all cases the degree of enrichment was determined by semiquantitative PCR assays of the bound and input DNA fractions following immunoprecipitation. The overall structural features of each gene category are summarized in diagrammatic form in Figure 2. These studies reveal a striking correlation between expression patterns and gene structure.

\section{GROUP I GENES}

Group I tissue-specific genes are essentially very similar to housekeeping genes. They are expressed in fibroblasts at relatively high levels, have an unmethylated $\mathrm{CpG}$ island promoter, and are packaged with an "open" nucleosome structure containing acetylated histones enriched for me-H3(K4) but depleted for me-H3(K9). These genes are considered tissue specific because they are expressed preferentially in one or a number of different cell types. Our studies clearly demonstrate that in terms of structure and expression, these genes are not repressed in the true sense of the word. Rather, they are packaged in an open nucleosome structure and their main
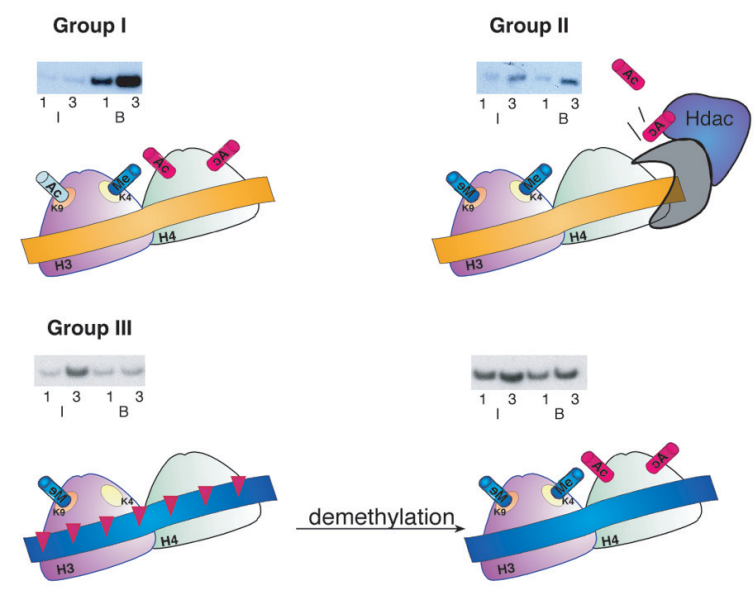

Group IV
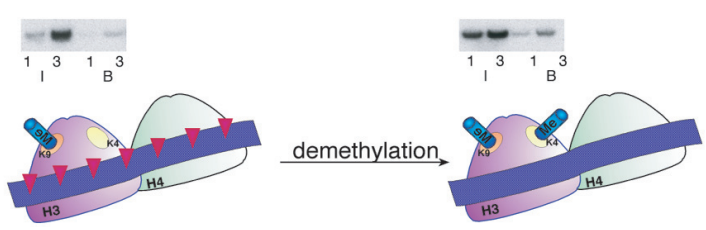

Figure 2. Epigenetic structures of tissue-specific genes. Histone modification patterns and DNA structure are shown for each category of tissue-specific genes. Group I and group II genes have $\mathrm{CpG}$ island (yellow ribbon) promoters that are constitutively unmethylated. Group I genes have acetylated histones and are methylated at H3(K4). ChIP analysis shows that the bound (B) fraction is enriched over the input (I) fraction. Group II genes are inhibited by repressors (gray) that recruit histone deacetylase (Hdac). H3(K4) remains methylated, and once deacetylated $\mathrm{H} 3$ (K9) can become methylated. Group III and group IV genes have non-CpG island (blue ribbon) promoters that are generally methylated (red triangles). In the presence of DNA methylation, histones are hypoacetylated (B is low as compared to I) and unmethylated at $\mathrm{H} 3(\mathrm{~K} 4)$. When the methyl groups are removed (Dnmt $\left.1^{-}\right)$, however, the histones undergo reacetylation and become methylated at $\mathrm{H} 3(\mathrm{~K} 4)$. For group IV genes, removal of methyl groups has no effect on histone acetylation (B is depleted as compared to I), but H3(K4) becomes methylated. Gene activity is not affected because these genes have an additional layer of repression that also works through histone acetylation.

mode of regulation is probably through interactions with tissue-specific transcriptional enhancers that boost their expression even more in the cell type of choice.

\section{GROUP II GENES}

Group II genes are normally expressed at low levels in fibroblasts, but can be readily induced simply by treating with TSA. Like group I genes, they contain CpG island promoter regions that are constitutively unmethylated, thus explaining why their level of transcription does not respond to undermethylation. In keeping with their repressed state, the promoter regions of these genes are uniformly packaged in nucleosomes containing deacetylated histones and me-H3(K9). In light of these structural details, it is very likely that these genes are inhibited by trans-acting repression factors, which work by bringing about local histone deacetylation. It is for this reason that transcription can be induced simply by reversing this 
state using TSA. In keeping with this idea, we found that many of the genes in this category are nervous tissue-specific and carry upstream cis-acting elements that bind the well characterized repressor, REST (Chong et al. 1995; Lunyak et al. 2002). This protein, which is expressed in all cell types except neurons, is known to work by recruiting histone deacetylases to the gene promoter (Huang et al. 1999).

\section{GROUP III GENES}

Almost all of the genes automatically induced in cells carrying the Dnmt1 knockout were indeed found to be methylated over their promoter sequences in normal fibroblasts. While these genes are underacetylated in wildtype cells, the removal of methyl groups converts nucleosomes at these promoters to an acetylated form. This is consistent with the idea that methylated DNA binds methyl-binding proteins, which then recruit histone deacetylases (Jones et al. 1998; Nan et al. 1998). Thus, once the methyl groups are removed, as in the Dnmt $1^{-}$ cells, these deacetylases will no longer be present at the promoter, allowing the DNA to get repackaged in an acetylated form. Since methyl-mediated repression is partially due to deacetylation (Eden et al. 1998), we find that TSA also has a small inductive effect on these genes. In addition to being packaged with underacetylated histones, these methylated genes also carry histones depleted for me-H3(K4). When methyl moieties are removed, however, the promoters get reassembled with histone $\mathrm{H} 3$ that is highly methylated at the lysine 4 position. Overall, it appears that genes in group III are repressed predominantly because of DNA methylation, and once this block is removed, the gene can now adopt an open chromatin conformation conducive to transcription.

It should be noted that some of the genes that are automatically induced in Dnmt1 ${ }^{-/}$cells actually have nonmethylated $\mathrm{CpG}$ island promoters (Jackson-Grusby et al. 2001) and appear to be constitutively packaged with acetylated histones $\mathrm{H} 3$ and H4 in wild-type cells. Although the mechanism for this repression has not been worked out, it should be noted that Dnmt1 itself has been shown to be capable of repressing transcription through a mechanism that is independent of DNA methylation (Fuks et al. 2000; Robertson et al. 2000). One possibility is that this protein recruits a histone methylase that modifies H3(K9) (Fuks et al. 2003), a mark that is clearly present on this category of genes.

\section{GROUP IV GENES}

The largest number of tissue-specific genes fall into group IV. These genes are expressed at extremely low levels and can be induced only by a combination of undermethylation and TSA treatment. Like group II genes, these sequences were found to be methylated in their promoter region and packaged in a closed nucleosome structure. In contrast to group III, however, group IV genes retain their closed nucleosome structure even after DNA methylation has been removed, suggesting that these genes are silenced by multiple layers of repression. Thus, while taking away DNA methylation would normally lead to an alteration in chromatin structure, these genes must be subject to additional repression mechanisms that also work by bringing about histone deacetylation.

It should be noted that induction of group IV genes by TSA in Dnmt1 ${ }^{-}$cells is only transient, and once the drug is removed, full gene repression is restored. This suggests that each layer of repression must be inherently programmed in the genome and is constantly capable of regenerating the closed deacetylated chromatin conformation even after nucleosomes have been artificially acetylated by TSA treatment. In other words, the histone acetylation pattern itself does not appear to be autonomously maintained.

\section{REPLICATION TIMING}

One potential candidate for another basic global repression mechanism is late replication timing. The genome as a whole is divided into replication time zones that are programmed to replicate at specific times in $\mathrm{S}$ phase (Goren and Cedar 2003). This pattern is an integral part of the overall chromosome structure characterized by a striking division into distinct structural and functional bands. At the level of individual genes, there appears to be a correlation between early replication and gene expression. Housekeeping genes all replicate early in $\mathrm{S}$ phase, while many tissue-specific genes are developmentally regulated to be late replicating in most tissues, but early replicating in their cell type of expression. Although this correlation has been known for a long time, it was only recently that experiments were done to test whether replication timing itself plays a role in the regulation of expression (Zhang et al. 2002).

Previous models had suggested that the transcriptional competence of each gene may actually be set up at the time of replication, while chromatin structure is somewhat relaxed. It was hypothesized that the factors necessary to impose gene activation could be cell cycle regulated and expressed only in early S (Gottesfeld and Bloomer 1982; Brown 1984). On the basis of this idea, we reasoned that naked DNA introduced into the nucleus early in S phase would become transcriptionally competent, while the same DNA would be inactive if introduced in late $\mathrm{S}$ cells. In order to test this simple concept, we inserted reporter genes into nuclei from early or late $\mathrm{S}$ phase using microinjection. To this end, Rat-1 cells were placed on slides carrying a well-marked grid that allowed each cell to be uniquely identified, and were then injected with reporters containing various different promoters linked to GFP or $\beta$-Gal coding sequences.

By following the division progress of each cell microscopically, it was possible to determine when in $\mathrm{S}$ phase each cell was injected (Fig. 3a). From a large series of these experiments using a wide variety of different promoters, we were able to show that plasmids injected in early $S$ nuclei are ten times more active than when the same plasmid was injected into late $\mathrm{S}$ phase cells (Zhang et al. 2002). Once set, this pattern remains fixed for a long time even though the cells themselves may continue cycling. Since this phenomenon was also observed with 
a.
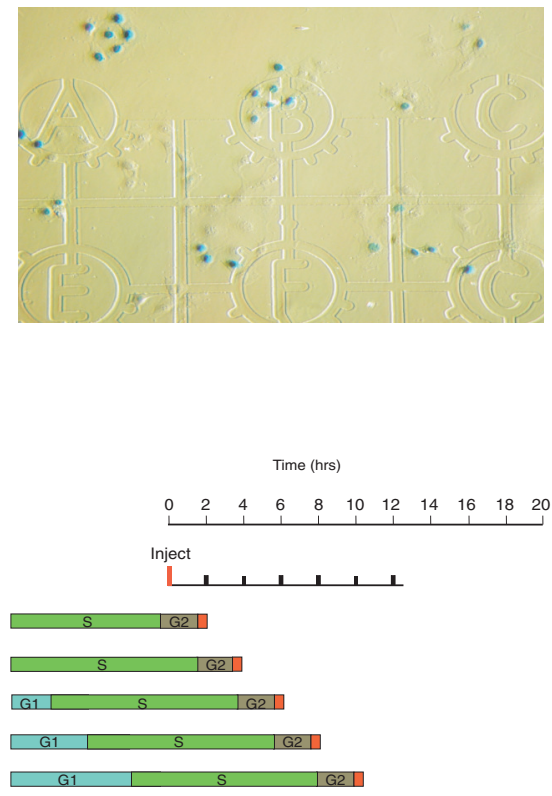

b.

$\beta$-actin
$\beta$-globin
Early
Late

Ac-H4

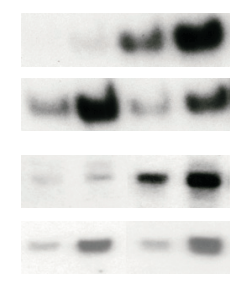

B
Ac-H3
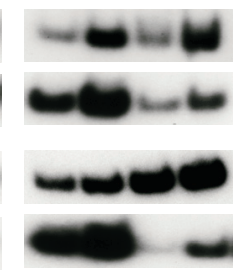

Figure 3. (a) Cells were plated on a Cellocate grid that contains markings that enable one to identify each cell in order to follow its cell cycle progression (Zhang et al. 2002). Cells were injected (red vertical bar) at zero time with a promoter-driven $\beta$-Gal reporter gene and followed every $2 \mathrm{hr}$ (short bars) to determine whether mitosis (red) had occurred. Mitosis at $10 \mathrm{hr}$ indicates that injection took place in early $\mathrm{S}$. Mitosis at $2 \mathrm{hr}$ indicates that injection took place in late $\mathrm{S}$. At $20 \mathrm{~h}$ after the injection, the cells were stained to visualize $\beta$-Gal, and the percentage of positive divided cells recorded. (b) Histone acetylation of early-replicating and late-replicating chromosomal Rat-1 cells injected in early $\mathrm{S}$ or late $\mathrm{S}$ with plasmid pBluescript and harvested $3 \mathrm{hr}$ later. ChIP (anti-Ac-H4 or anti-Ac-H3) was performed on mononucleosomes and enrichment was determined for total plasmid sequences (which should be the same in both cell populations), as well as for $\beta$-actin and $\beta$-globin (Zhang et al. 2002). Note that early injected plasmids are enriched for acetylation while late injected DNA is depleted.

replicating plasmids as well, these results strongly suggest that late replication may indeed represent an endogenous mechanism for repressing gene expression.

In order to understand the molecular mechanism of this effect, we next asked whether transcriptional competence may be set up through effects on nucleosome structure. To this end, we injected plasmids into either early or late $\mathrm{S}$ phase cells and then used ChIP to determine their histone modification pattern. As shown in Figure 3b, plasmids injected into early $\mathrm{S}$ phase cells are highly enriched in nucleosomes containing acetylated histones $\mathrm{H} 3$ and
H4, while late-injected plasmids are automatically packaged with histones depleted for acetylation.

These studies provide an elementary picture of how replication timing may play a role in regulating gene expression. According to this model, replication timing is a basic property of each chromosomal band that is set up during early development in the embryo. Once established, each region replicates at a fixed time in S phase in every division cycle. As part of the process of DNA replication, nucleosomal structure becomes disrupted. Once the replication fork has passed by, this structure must then be restored. If replication takes place in early $\mathrm{S}$, the newly synthesized DNA will be assembled with nucleosomes containing acetylated histones. On the other hand, genes replicating in late $\mathrm{S}$ will get packaged with deacetylated histones. Although the precise mechanism for this is not known, it has been shown that late replication foci are specifically associated with histone deacetylase 2 (Hdac2) (Rountree et al. 2000), and this could clearly serve as a basis for late $\mathrm{S}$ repression. In this scheme, replication timing serves as a global mechanism for maintaining gene expression patterns through cell division.

\section{REPRESSION BY LATE REPLICATION}

With this picture in mind, we then asked whether late replication timing may represent one of the mechanisms involved in the repression of group IV genes. To this end, we used fluorescent in situ hybridization (FISH) to assay the replication timing pattern (Selig et al. 1992) of genes in each tissue-specific expression category. In this procedure, bacterial artificial chromosome (BAC) clones containing each individual gene sequence are labeled with biotinylated nucleotides and used as hybridization probes on interphase wild-type fibroblast nuclei labeled with BrdU. Each S-phase nucleus (BrdU positive) reveals either two single hybridization dots if the gene has not yet undergone replication, or two double dots if it has. By counting the number of nuclei containing single or double signals one can calculate the replication time. Genes that yield a relatively high number of double dots must replicate early in $\mathrm{S}$, while genes showing a relatively high number of singles replicate in late S (Fig. 4a).

Using this method, we obtained a full snapshot of replication timing in the genome (Figs. $4 \mathrm{~b}$ and 5). All of the genes in groups I, II, and III appear to replicate in early S. The significance of this is that these genes would have a normal tendency to be packaged with acetylated histones following replication. In the case of group II, the presence of repressor proteins would counteract this by actively causing local deacetylation. In a similar manner, group III genes would become deacetylated by virtue of the presence of DNA methylation. Since group I genes do not have any inherent repression mechanisms based on histone deacetylation, they would retain the acetylation pattern that is predetermined by early replication.

\section{MULTIPLE LAYERS OF REPRESSION}

As predicted from our model, $>60 \%$ of all group IV genes replicate late in S phase, and thus could represent 
a.

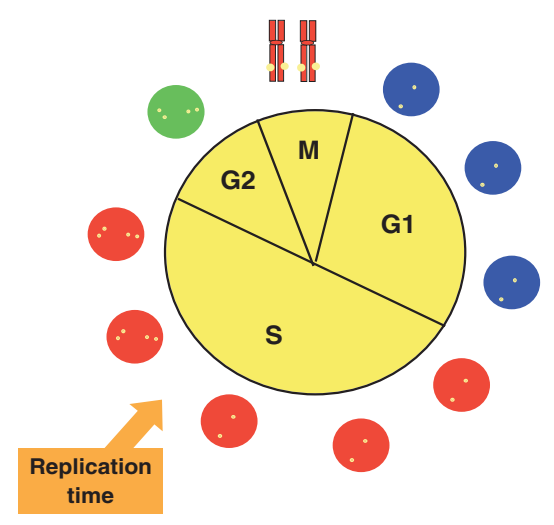

b.

\begin{tabular}{|c|c|c|}
\hline Group & Gene Name & $\%$ singles \\
\hline \multirow{2}{*}{ HK } & $\beta$-actin & $34(\mathrm{E})$ \\
\hline & Dhfr & $38(E)$ \\
\hline \multirow{2}{*}{ I } & Mea & $37(E)$ \\
\hline & $\overline{C D 24}$ & $38(E)$ \\
\hline II & Int-1 & $46(E)$ \\
\hline \multirow{2}{*}{ III } & KC & $39(E)$ \\
\hline & Pem & $36(E)$ \\
\hline \multirow{4}{*}{ IV } & Oct-4 & $37(E)$ \\
\hline & Y1-globin & $73(\mathrm{~L})$ \\
\hline & $\beta$-globin & $64(\mathrm{~L})$ \\
\hline & Thy 19.4 & 66 (L) \\
\hline
\end{tabular}

Figure 4. (a) How gene sequences are detected by FISH throughout the cell cycle. Each allele is represented by a single dot. In G1 prior to replication, each homologous chromosome generates a single dot. In S phase, until the gene replicates there are two single dots. After the specific gene has replicated, however, one will observe two sets of double dots. The time point where the transition from two single dots to two doublets occurs depends on the replication time of each gene. In G2 all genes have two sets of double dots. In metaphase there are two chromosomes, each composed of two sister chromatids, so that they yield four dots altogether. $(b)$ Representable examples from the four gene groups, assayed for their replication timing by FISH (Selig et al. 1992). Replication time is shown as percentage of single dots. Genes with $\leq 50 \%$ singlets are early replicating, whereas genes whose percentage of single dots is more than 60 are late replicating.

one of the multiple mechanisms employed for gene repression. Since these genes are also methylated, they carry not one, but two fundamental repression layers. Thus, when DNA methylation is removed (Dnmt1 ${ }^{-}$cells), methyl groups are no longer present to recruit deacetylase activity, but the gene promoters remain unacetylated by virtue of late replication timing, which automatically packages this DNA in a closed conformation following replication. It should be noted that some group IV genes do not replicate in late $\mathrm{S}$ and thus must have additional layers of repression that have not yet been characterized. One possibility is that these genes, like those in group II,

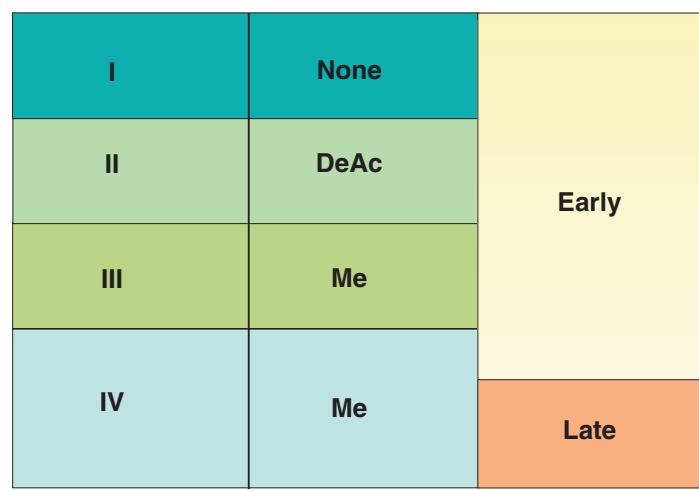

Figure 5. Summary of the mechanisms responsible for the repression status of tissue-specific genes. Group I genes are not repressed and have an open chromatin structure. Group II genes are probably repressed by trans-acting repressors and are therefore packed in a closed chromatin conformation. Group III genes are repressed by DNA methylation. All of these groups replicate early in the cell cycle. Group IV genes are repressed by DNA methylation and by additional mechanisms that serve to maintain the repressed state even after the methyl moieties have been removed. Late replication timing may represent one of these effectors. Many of these genes are late replicating, and this could explain the layered effect. Other genes in group IV may be influenced both by methylation and by specific repressors, as in group II.

are subject to factor-mediated repression. Thus, these genes would also have two independent mechanisms for packaging genes in a closed nucleosome structure, DNA methylation (like group III) and transcriptional repressors capable of recruiting histone deacetylases (like group II). One example of this is Oct4, which has been shown to be inactivated by both DNA methylation (Gidekel and Bergman 2002) and, more recently, Polycomb-mediated repression (N. Feldman, unpubl.).

\section{HISTONE CODE HYPOTHESIS}

It has been suggested that modifications on histone tail lysines and arginines are used as a molecular code for affecting nucleosome structure and in this way regulate different expression paradigms (Jenuwein and Allis 2001). Currently, most of the data describing histone modification states for different gene sequences reveal a more conservative picture where active genes are always packaged with nucleosomes containing histones that are generally acetylated and methylated at $\mathrm{H} 3(\mathrm{~K} 4)$. In the same manner, inactive genes are also characterized by a standard structure in which the "activating modifications" are absent, but H3 is methylated on lysine 9 (Vermaak et al. 2003).

In our study we demonstrate for the first time that there is indeed a code that relates epigenetic mechanisms at the level of DNA to nucleosome structure. The most striking example of this is the effect of DNA methylation on me$\mathrm{H} 3(\mathrm{~K} 4)$. Genes that contain unmethylated $\mathrm{CpG}$ island promoters are all packaged with histone $\mathrm{H} 3$ methylated at lysine 4 regardless of the activity state of the gene or the level of histone acetylation. In contrast, this lysine residue is always unmodified in nucleosomes covering methylated DNA. Removal of DNA methylation appears to bring about an automatic reversal that allows this ly- 
sine 4 to become methylated. Although the mechanism for this phenomenon has not yet been elucidated, it is reasonable to assume that the presence of methyl moieties on the DNA inhibits histone H3(K4) methylation. It should be noted that in our studies, H3(K9) methylation also seems to behave like an independent variable with many genes remaining modified at this position even within the context of general histone acetylation. Although our experiments do not reveal the role of each histone modification in the regulation of gene expression, they do indicate that these modifications help to define different gene repression paradigms.

\section{STEPWISE GENE ACTIVATION}

The fact that many tissue-specific genes are repressed by multiple layers of gene repression has a significant influence on the way in which these genes ultimately become activated in their cell type of expression. While group I genes can evidently be induced automatically once they are exposed to tissue-specific trans activators, all of the other groups are probably packaged in an inaccessible form that prevents these interactions, and this structure must be opened before high level expression can be attained. A good example of how this may occur is the human $\beta$-globin gene locus on chromosome 11 in man. This region contains three globin genes $(\varepsilon, \gamma, \beta)$, which are specifically expressed in different cell types during development (Fu et al. 2002).

In nonexpressing tissues, the locus is late replicating and is in a DNaseI-insensitive chromatin conformation with all of the gene promoters being methylated. In all erythroid cells, the first change in structure occurs early in the differentiation process when the entire globin region becomes early replicating (Epner et al. 1988; Simon et al. 2001) and DNaseI sensitive (Forrester et al. 1986). Despite this overall partial opening, only one of the genes actually undergoes demethylation and becomes active (Busslinger et al. 1983). This could be the $\varepsilon$ gene in embryonic blood, the $\gamma$ gene in fetal liver, or the $\beta$ gene in adult erythroid precursors. It appears that only the demethylated gene is fully accessible to tissue-specific trans-acting factors. While the other genes in the cluster have already shed some of their epigenetic repression mechanisms, they still remain inactive because of the closed structure imposed on them by the presence of DNA methylation. In the immune system, as well, repression is removed in a stagewise manner with demethylation being required as the last step that makes the locus accessible to the rearrangement machinery of the cell (Bergman and Cedar 2004).

\section{ACKNOWLEDGMENTS}

This work was supported by grants from the National Institutes of Health, the Israel Science Foundation, the Israel Cancer Research Fund, and the Belfer Foundation.

\section{REFERENCES}

Bergman Y. and Cedar H. 2004. A stepwise epigenetic process controls immunoglobulin allelic exclusion. Nat. Rev. Im- munol. 4: 753.

Brandeis M., Frank D., Keshet I., Siegfried Z., Mendelsohn M., Nemes A., Temper V., Razin A., and Cedar H. 1994. Sp1 elements protect a $\mathrm{CpG}$ island from de novo methylation. $\mathrm{Na}$ ture 371: 435.

Brown D.D. 1984. The role of stable complexes that repress and activate eucaryotic genes. Cell 37: 359 .

Busslinger M., Hurst J., and Flavell R.A. 1983. DNA methylation and the regulation of the globin gene expression. Cell 34: 197.

Cedar H. 1988. DNA methylation and gene activity. Cell 53: 3.

Chong J.A., Tapia-Ramirez J., Kim S., Toledo-Aral J.J., Zheng Y., Boutros M.C., Altshuller Y.M., Frohman M.A., Kraner S.D., and Mandel G. 1995. REST: A mammalian silencer protein that restricts sodium channel gene expression to neurons. Cell 80: 949 .

Eden S., Hashimshony T., Keshet I., Thorne A.W., and Cedar H. 1998. DNA methylation models histone acetylation. Nature 394: 842 .

Epner E., Forrester W.C., and Groudine M. 1988. Asynchronous DNA replication within the human $\beta$-globin gene locus. Proc. Natl. Acad. Sci. 85: 8081.

Forrester W.C., Thompson C., Elder J.T., and Groudine M. 1986. A developmentally stable chromatin structure in the human beta-globin gene cluster. Proc. Natl. Acad. Sci. 83: 1359.

Fu X.H., Liu D.P., and Liang C.C. 2002. Chromatin structure and transcriptional regulation of the beta-globin locus. Exp. Cell Res. 278: 1.

Fuks F., Hurd P.J., Deplus R., and Kouzarides T. 2003. The DNA methyltransferases associate with HP1 and the SUV39H1 histone methyltransferase. Nucleic Acids Res. 31: 2305.

Fuks F., Burgers W.A., Brehm A., Hughes-Davies L., and Kouzarides T. 2000. DNA methyltransferase Dnmt1 associates with histone deacetylase activity. Nat. Genet. 24: 88.

Gidekel S. and Bergman Y. 2002. A unique developmental pattern of Oct-3/4 DNA methylation is controlled by a cis-demodification element. J. Biol. Chem. 277: 34521.

Goren A. and Cedar H. 2003. Replicating by the clock. Nat. Rev. Mol. Cell Biol. 4: 25.

Gottesfeld J. and Bloomer L.S. 1982. Assembly of transcriptionally active 5S RNA gene chromatin in vitro. Cell 28: 781 .

Gruenbaum Y., Stein R., Cedar H., and Razin A. 1981. Methylation of CpG sequences in eukaryotic DNA. FEBS Lett. 123: 67.

Hashimshony T., Zhang J., Keshet I., Bustin M., and Cedar H. 2003. The role of DNA methylation in setting up chromatin structure during development. Nat. Genet. 34: 187.

Huang Y., Myers S.J., and Dingledine R. 1999. Transcriptional repression by REST: Recruitment of Sin3A and histone deacetylase to neuronal genes. Nat. Neurosci. 2: 867.

Jackson-Grusby L., Beard C., Possemato R., Tudor M., Fambrough D., Csankovszki G., Dausman J., Lee P., Wilson C., Lander E., and Jaenisch R. 2001. Loss of genomic methylation causes $\mathrm{p} 53$-dependent apoptosis and epigenetic deregulation. Nat. Genet. 27: 31.

Jenuwein T. and Allis C.D. 2001. Translating the histone code. Science 293: 1074

Jones P.L., Veenstra G.J.C., Wade P.A., Vermaak D., Kass S.U., Landsberg N., Strouboulis J., and Wolffe A.P. 1998. Methylated DNA and MeCP2 recruit histone deacetylase to repress transcription. Nat. Genet. 19: 187.

Kafri T., Ariel M., Brandeis M., Shemer R., Urven L., McCarrey J., Cedar H., and Razin A. 1992. Developmental pattern of gene-specific DNA methylation in the mouse embryo and germline. Genes Dev. 6: 705.

Kuhn R., Schwenk F., Aguet M., and Rajewsky K. 1995. Inducible gene targeting in mice. Science 269: 1427.

Lallemand Y., Luria V., Haffner-Krausz R., and Lonai P. 1998. Maternally expressed PGK-Cre transgene as a tool for early and uniform activation of the Cre site specific recombinase. Transgenic Res. 7: 105.

Li E., Bestor T.H., and Jaenisch R. 1992. Targeted mutation of the DNA methyltransferase gene results in embryonic lethality. Cell 69: 915.

Lunyak V.V., Burgess R., Prefontaine G.G., Nelson C., Sze 
S.-H., Chenoweth J., Schwartz P., Pevzner P.A., Glass C., Mandel G., and Rosenfeld M.G. 2002. Corepressor-dependent silencing of chromosomal regions encoding neuronal genes. Science 298: 1747.

Macleod D., Charlton J., Mullins J., and Bird A.P. 1994. Sp1 sites in the mouse Aprt gene promoter are required to prevent methylation of the CpG island. Genes Dev. 8: 2282.

Nan X., Ng H.-H., Johnson C.A., Laherty C.D., Turner B.M., Eisenman R.N., and Bird A. 1998. Transcriptional repression by the methyl-CpG-binding protein $\mathrm{MeCP} 2$ involves a histone deacetylase complex. Nature 393: 386.

Okano M., Bell D.W., Haber D.A., and Li E. 1999. DNA methyltransferases Dnmt3a and Dnmt3b are essential for de novo methylation and mammalian development. Cell 99: 247.

Razin A. 1998. CpG methylation, chromatin structure and gene silencing-A three-way connection. EMBO J. 17: 4905.

Reynaud C., Bruno C., Boullanger P., Grange J., Barbesti S., and Niveleau A. 1992. Monitoring of urinary excretion of modified nucleosides in cancer patients using a set of six monoclonal antibodies. Cancer Lett. 61: 255.

Robertson K.D., Ait-Si-Ali S., Yokochi T., Wade P.A., Jones
P.L., and Wolffe A.P. 2000. DNMT1 forms a complex with $\mathrm{Rb}, \mathrm{E} 2 \mathrm{~F} 1$ and $\mathrm{HDAC} 1$ and represses transcription from E2Fresponsive promoters. Nat. Genet. 25: 338 .

Rountree M.R., Bachman K.E., and Baylin S.B. 2000. DNMT1 binds HDAC2 and a new co-repressor, DMAP1, to form a complex at replication foci. Nat. Genet. 25: 269.

Selig S., Okumura K., Ward D.C., and Cedar H. 1992. Delineation of DNA replication time zones by fluorescence in situ hybridization. EMBO J. 11: 1217.

Siegfried Z., Eden S., Mendelsohn M., Feng X., Tzubari B., and Cedar H. 1999. DNA methylation represses transcription in vivo. Nat. Genet. 22: 203.

Simon I., Tenzen T., Mostoslavsky R., Fibach E., Lande L., Milot E., Gribnau J., Grosveld F., Fraser P., and Cedar H. 2001. Developmental regulation of DNA replication timing at the human $\beta$ globin locus. EMBO J. 20: 6150.

Vermaak D., Ahmad K., and Henikoff S. 2003. Maintenance of chromatin states: An open-and-shut case. Curr. Opin. Cell Biol. 15: 266.

Zhang J., Feng X., Hashimshony T., Keshet I., and Cedar H. 2002. The establishment of transcriptional competence in early and late S-phase. Nature 420: 198. 


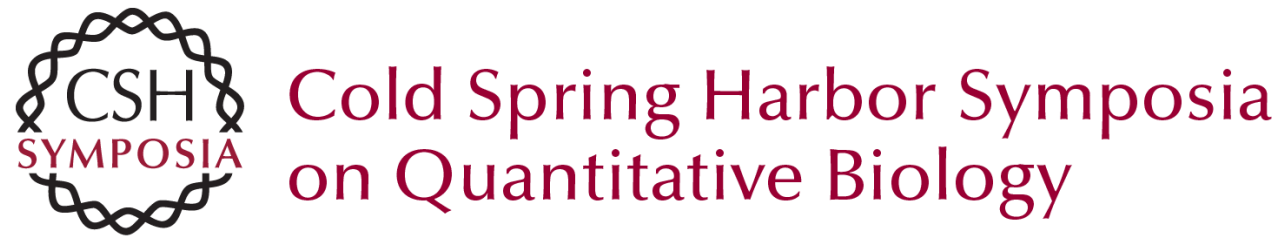

\section{Gene Repression Paradigms in Animal Cells}

L. LANDE-DINER, J. ZHANG, T. HASHIMSHONY, et al.

Cold Spring Harb Symp Quant Biol 2004 69: 131-138

Access the most recent version at doi:10.1101/sqb.2004.69.131

References This article cites 38 articles, 10 of which can be accessed free at: http://symposium.cshlp.org/content/69/131.full.html\#ref-list-1

\section{License}

Email Alerting Receive free email alerts when new articles cite this article - sign up in Service the box at the top right corner of the article or click here. 